\title{
PROBLEMÁTICA DE LA GANADERÍA EXTENSIVA EN ESPAÑA
}

\author{
POR \\ CARLOS J. PARDO ABAD
}

\section{Introducción}

El estudio de la ganadería extensiva es interesante desde diversos puntos de vista, pero particularmente desde el geográfico por varias razones. En primer lugar, porque la ganadería extensiva, tras un proceso de constante intensificación ganadera que ha perseguido alcanzar la máxima producción, ha quedado totalmente marginada y afectada desde mediados del siglo actual por una profunda crisis. En segundo lugar, porque en este tema confluyen aspectos de indudable carácter geográfico, como la puesta en valor de extensos territorios o la utilización de unos recursos naturales que de otra forma quedarian desaprovechados. $\mathrm{Y}$ en tercer lugar, porque permite relacionar aspectos muy diversos de la realidad; dentro de la Geografía Agraria, la ganadería extensiva es quizá el tema en el que menos se ha profundizado, siendo, sin embargo, uno de los más motivadores.

La tendencia intensificadora que ha invadido las explotaciones pecuarias ha motivado en los sistemas extensivos una serie de obligadas reacciones para dar respuesta a las nuevas exigencias planteadas, cuando no la crisis definitiva. Para ello se ha requerido aumentar los rendimientos alimenticios del suelo, bien con aportes 
energéticos complementarios (abonos) o con métodos más racionales de pastoreo, así como el cruce genético para obtener mejores niveles de producción o la suplementación alimenticia a base de concentrados. Esta evolución, aunque muy tímida en bastantes ocasiones, ha incrementado las cargas ganaderas, la tecnificación y una mayor imbricación con el mercado. En líneas generales, sin embargo, el ciclo energético es muy cerrado y sólo en los casos más evolucionados asistimos a diversas rupturas del ciclo por la compra de recursos exteriores en forma de abonos, alimentos, etc.

El desenlace de la crisis que vive la ganadería extensiva, y particularmente en España, parece difícil. La alternativa mayoritaria ha sido la intensificación, cuando no el abandono definitivo de la ganadería. Esta reestructuración no significa la solución a todos los problemas: la transformación de carácter industrial ha empeorado, incluso, las perspectivas del subsector. Sólo una política imaginativa de ayuda, fomento y potenciación de los recursos propios, procurando al mismo tiempo la existencia de unidades de dimensiones aceptables, puede convertir al régimen extensivo en plenamente viable y vincularlo definitivamente a las exigencias actuales.

\section{Variabilidad de las áreas de aprovechamiento ganadero}

La ganadería extensiva se corresponde con un sistema de producción basado en las variabilidades naturales y en la existencia de una base territorial concreta, descansando en el aprovechamiento de los pastos. La racionalidad en el uso de las especies pratenses asegura, por tanto, una mejor alimentación del ganado y la pervivencia de las plantas herbáceas.

La ordenación en el uso de los pastos es fundamental. El objetivo perseguido es, por un lado, aumentar los rendimientos ganaderos y, por otro, conservar los recursos naturales. El sistema tradicional de pastoreo ha sido el continuo o indiscriminado, dejando que el ganado comiera la hierba libremente durante un cierto período de tiempo. Como de esta forma se desperdician grandes cantidades de pastos o éstos no se consumen en el momento óptimo, ha sido sustituido progresivamente por otros sistemas de pastoreo, como el diferido, que 
significa un retraso en el comienzo del aprovechamiento del pastizal hasta el momento en que las especies pratenses hayan alcanzado cierto crecimiento y desarrollo. Para ello suele dejarse una parcela cada año libre de pastoreo para permitir un mejor agarre de las plantas. A veces también se ha introducido el llamado pastoreo rotacional, que consiste en dividir la superficie pastable en un determinado número de parcelas, pasando los animales de una a otra mediante una rotación.

Estos nuevos sistemas han revolucionado en cierto sentido las explotaciones ganaderas extensivas, variando los esquemas de producción, aumentando la productividad y reduciendo los costes de la actividad. Sin embargo, las ventajas de estos sistemas parecen corresponderse más con los pastos de climas atlánticos que con los mediterráneos, debido a que disfrutan de altas cargas ganaderas y elevados rendimientos.

Ha sido tradicional en España, y en toda la cuenca mediterránea, el pastoreo integrado con actividades agrícolas y forestales, es decir, el pastoreo del ganado sobre eriales, baldíos, montes leñosos y huecos, rastrojeras, barbechos, etc. Esta integración ha permitido un mayor uso agrario del terreno, creando una compleja diversidad de áreas de aprovechamiento ganadero. La superficie pastable total de España ascendía a comienzos de los años noventa a 23.079 .800 ha., incluyendo prados naturales, pastizales, eriales a pastos, barbechos y montes huecos y leñosos. Esta cifra representa, aproximadamente, el $45 \%$ de la superficie geográfica total. Se ha prescindido de las rastrojeras pastadas debido a que su capacidad de sostenimiento ganadero es mínima, así como bastante variable y difícil de establecer; y de igual forma, habría que restar lo no pastable de pastizales, eriales, etc.

La evolución de la superficie pastable desde 1975 a 1990 permite observar un descenso cifrado en 1.153 .600 ha. (-5 \%). Esto contrasta con el aumento de la extensión de tierras cultivadas en regadío, que pasa de 2.616.800 ha. en 1975 a 3.136 .800 en 1988, es decir, se produce un incremento total en dicho período de más de medio millón de hectáreas.

La mayor superficie de aprovechamiento ganadero en nuestro país la representan los pastizales, que se diferencian de los prados 
EVOLUCIÓN DE LA SUPERFICIE PASTABLE EN ESPAÑA

\begin{tabular}{lccc} 
Año & Superficie (ha.) & Año & Superficie (ha.) \\
\hline 1975 & 24.233 .400 & 1988 & 23.269 .100 \\
1980 & 24.060 .200 & 1989 & 23.126 .800 \\
1985 & 23.231 .200 & 1990 & 23.079 .800
\end{tabular}

FuEntE: MAPA, Anuario de Estadistica Agraria, varios años.

naturales porque se dan en climas más secos y no pueden ser aprovechados por siega. Su superficie supera los cinco millones de hectáreas, muy por encima de los prados naturales. Estos últimos son la menor superficie pastable en España. El resto de las áreas, es decir, los eriales a pastos, los montes abiertos y leñosos y los barbechos, disponen de superficies muy parecidas, destacando muy ligeramente la categoría de monte leñoso. En conjunto, se puede afirmar que una buena parte de la superficie española total está potencialmente destinada a producir recursos alimenticios para la ganadería.

Las mayores superficies pastables se localizan en las regiones de Castilla y León, Galicia, Aragón, Castilla-La Mancha, Extremadura y Andalucía, con cifras muy superiores en algunos casos a los tres millones de hectáreas. Pero las cifras absolutas están en relación, como es lógico, con la propia superficie de cada una de las Comunidades Autónomas. Más significativo es ver qué especialización presentan las distintas regiones o zonas del país en cada tipo de superficie pastable. Las diferencias internas permiten establecer lo que hemos denominado dominios preferentes de aprovechamiento ganadero en España (figura 1) y realizados eligiendo para cada provincia la superficie pastable con mayor número de hectáreas. En el norte dominan los prados naturales, los pastizales en las áreas de montaña, el monte abierto en el suroeste, los barbechos en las dos mesetas y en la parte central del valle del Ebro y el monte leñoso en la vertiente mediterránea. Se configura, por tanto, un amplio y variado abanido de posibilidades para la explotación ganadera y la utilización de los recursos naturales, lo que contrasta con la evolución decreciente y la crisis por la que atraviesa en estos momentos el aprovechamiento extensivo.

$$
-128-
$$


SUPERFICIE DE LAS ÁREAS DE APROVECHAMIENTO GANADERO EN ESPAÑA. 1990

\begin{tabular}{|c|c|c|}
\hline Área & Superficie (miles ha.) & $\%$ \\
\hline Prados naturales & $1.389,7$ & 6,0 \\
\hline Pastizales ................................. & $5.368,3$ & 23,2 \\
\hline Eriales a pastos & $3.541,7$ & 15,3 \\
\hline 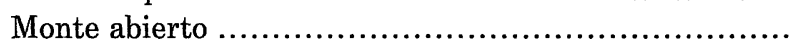 & $3.636,4$ & 15,8 \\
\hline 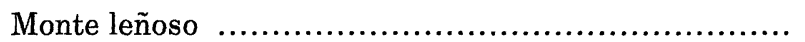 & $4.981,4$ & 21,6 \\
\hline Barbechos & $4.162,3$ & 18,1 \\
\hline 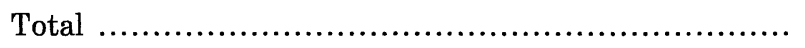 & $23.079,8$ & 100 \\
\hline
\end{tabular}

Fuente: MAPA, Anuario de Estadística Agraria, 1990, y elaboración propia.

Cuadro III

SUPERFICIES PASTABLES POR CC.AA. EN 1990 (MILES DE HA.)

\begin{tabular}{|c|c|c|c|c|c|c|}
\hline CC.AA. & $\begin{array}{c}\text { Prados } \\
\text { naturales }\end{array}$ & $\begin{array}{l}\text { Pasti- } \\
\text { zales }\end{array}$ & $\begin{array}{l}\text { Erial a } \\
\text { pastos }\end{array}$ & $\begin{array}{l}\text { Mte. } \\
\text { abierto }\end{array}$ & $\begin{array}{c}\text { Mte. } \\
\text { leñoso }\end{array}$ & $\begin{array}{c}\text { Barbe- } \\
\text { chos }\end{array}$ \\
\hline Galicia ... & 206,9 & 147,3 & 12,5 & 242,6 & 764,7 & 32,6 \\
\hline Asturias ................... & 210,5 & 72,6 & 164,7 & - & 225,7 & . \\
\hline Cantabria .................. & 137,0 & 11,7 & 42,2 & 76,2 & 52,4 & 0,2 \\
\hline P. Vasco .................... & 90,3 & 26,9 & 13,4 & 23,9 & 75,5 & 2,6 \\
\hline Castilla y León ........... & 419,6 & $1.200,8$ & 829,2 & 718,7 & 686,9 & 886,3 \\
\hline Madrid .................... & 48,2 & 86,9 & 84,6 & 69,0 & 41,3 & 93,0 \\
\hline La Rioja .................. & 13,0 & 115,6 & 53,2 & 14,6 & 36,4 & 18,8 \\
\hline Navarra . & 61,2 & 225,6 & 35,5 & 13,7 & 77,8 & 62,1 \\
\hline Aragón ..................... & 41,7 & 713,5 & 456,9 & 179,0 & 467,0 & 558,9 \\
\hline Cataluña ................... & 50,1 & 332,0 & 90,8 & 24,4 & 361,9 & 62,1 \\
\hline Comunidad Valenciana . & 1,4 & 25,1 & 198,6 & 132,5 & 436,2 & 170,2 \\
\hline Castilla La Mancha ...... & 37,3 & 697,3 & 593,2 & 410,8 & 625,6 & $1.021,4$ \\
\hline Extremadura .............. & 58,9 & 905,9 & 185,2 & 725,4 & 305,7 & 413,6 \\
\hline Andalucía ................. & 12,8 & 746,6 & 500,2 & 891,4 & 653,4 & 545,2 \\
\hline Murcia .................... & - & 21,6 & 58,0 & 78,2 & 109,4 & 185,9 \\
\hline Baleares .................. & - & - & 20,0 & 34,1 & 53,2 & 35,5 \\
\hline 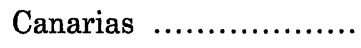 & - & 38,1 & 202,5 & 1,2 & 7,4 & 73,4 \\
\hline Total ......................... & $1.389,7$ & $5.368,3$ & $3.541,7$ & $3.636,4$ & $4.981,4$ & $4.162,3$ \\
\hline
\end{tabular}

Fuente: MAPA, Anuario de Estadistica Agraria. 1990. 


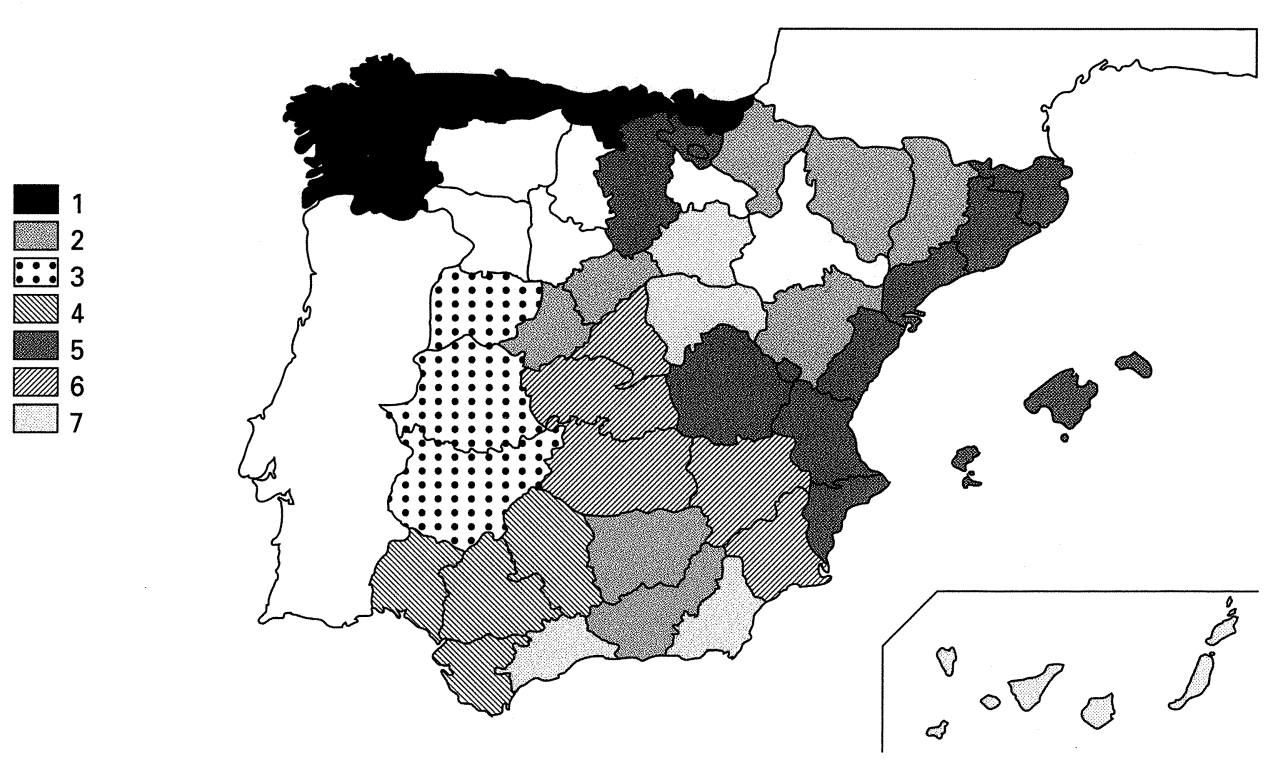

FIGURA 1.-Dominios preferentes de aprovechamiento ganadero en España. 1. Prados naturales y monte leñoso; 2 . pastizales; 3. montes abiertos y pastizales; 4. monte abierto; 5. monte leñoso; 6 . barbechos; 7. eriales a pastos.

La dehesa (que en Portugal recibe el nombre de «montado») es un área importante de aprovechamiento ganadero de los recursos naturales, utilizados tradicionalmente por una serie de razas autóctonas bien adaptadas a este ecosistema, que domina en todo el suroeste peninsular, estimándose para la parte española en unas 800.000 cabezas de vacuno, 4,7 millones de ovino, 760.000 de caprino y 760.000 de porcino.

Aunque los pastos son variados, presentan un carácter anual y son escasas las especies perennes. El incremento de la producción y, por tanto, de la carga ganadera, depende de la fertilización, de la introducción de especies nuevas y del uso del pastoreo ligeramente diferido. Estas mejoras podrían aumentar la carga de las dehesas y adecuar la producción a los valores medios nacionales, ya que aún se sitúa aproximadamente en torno a la mitad. Si la producción media de carne de vacuno en la dehesa es de 87 kilos por vaca, en el conjunto de España esta cifra se eleva a 160,8 kilos (Martín Bellido, 1989). 
Las diferencias de clima y relieve entre unas regiones y otras han motivado desde antiguo la aparición de una práctica íntimamente relacionada con la ganadería extensiva: la trashumancia. La trashumancia, que en definitiva es una modalidad de pastoreo estacional, subsana la variabilidad de los pastos disponibles para el ganado, desplazando el rebaño (frecuentemente ovino) a los recursos naturales. La crisis por la que atraviesa la ganadería extensiva ha repercutido de manera notable en el pastoreo trashumante, afectando particularmente a los desplazamientos de largo recorrido. En ello ha jugado un papel importante la reducción en muchas zonas del ganado ovino, pero también las insuficientes atenciones prestadas por la Administración a un método considerado arcaico y poco renovado.

Los factores geográficos han condicionado desde siempre esta práctica en España (contrastes climáticos, difícil orografía, oscilaciones térmicas, etc.); pero también hay que destacar los de tipo histórico, relacionándose en nuestro país con la propia evolución política y económica. El pastoreo trashumante se desarrolla plenamente a partir del establecimiento de la Mesta, al amparo que una política protectora hacia la ganadería extensiva que concebía la riqueza pecuaria como eje fundamental de la vida económica y social de la Corona. En 1836 desaparece la Mesta y ello se inscribe en un contexto general de liberalización de las actividades agrarias y de implantación de un marco jurídico de tipo burgués.

Pero, realmente, la decadencia de la ganadería extensiva y, con ello, de la trashumancia, se produce a partir de 1950. En la actualidad, esta práctica se encuentra en declive y ha variado desde una trashumancia generalizada a una de tipo más restringido y puntual y limitada a ciertos casos concretos. Algunos estudios han calculado que la trashumancia era practicada a comienzos de los años ochenta aproximadamente por unas 500.000 cabezas de ovino en España. La decadencia es evidente si se compara con momentos anteriores, pero aún se siguen buscando las facilidades climáticas que favorecen un mejor desarrollo de los recursos naturales renovables.

Las cañadas, o vías de comunicación ganadera, constituyen la infraestructura básica de la trashumancia en busca de pastos complementarios. Con diferentes denominaciones según las regiones («caba- 
ñeras» en Aragón, «azadores reales» en Valencia, «cañadas» en Navarra, «cañadas reales» en Castilla y «carrerades» en Cataluña), esta red pecuaria atraviesa la Península Ibérica mediante un trazado preferentemente vertical, que enlaza las zonas montañosas de la submeseta norte con los pastizales de invierno de Andalucía, Extremadura y La Mancha.

El abandono progresivo de la ganadería extensiva ha degradado estas vías pecuarias, sobre todo si se tiene en cuenta la competencia ejercida por el ferrocarril en los desplazamientos de ganado y las numerosas privatizaciones y rompimientos de la trama cañariega. Algunas partes de cañadas han sido roturadas y otras agregadas a propiedades colindantes. La culminación se puede encontrar en la Ley de Vías Pecuarias de 22 de junio de 1974, que permitía desamortizar cañadas a los particulares próximos o a organismos de la Administración, siempre que existiera un «beneficio» público y fines utilitarios distintos a los pecuarios.

De lo aproximadamente 125.000 kilómetros de cañadas en toda la Península Ibérica (García Martín, 1990), sólo una parte reducida, la correspondiente a las áreas serranas más tradicionales, sigue con cierta utilidad hasta los puntos de embarque de ferrocarril o camiones. Los viejos modos de trashumar el ganado con largos recorridos son cada vez más excepcionales y han sido sustituidos o por desplazamientos cortos (trasterminancias) o por el uso de nuevos medios de transporte, fundamentalmente trenes y camiones, que han reemplazado algunos de los itinerarios tradicionales. Sin embargo, las áreas de origen y destino de los rebaños para el aprovechamiento de los pastos se mantienen con pocas variaciones.

La trashumancia constituye, en definitiva, una práctica rentable al mantener en activo territorios generalmente poco productivos $\mathrm{y}$ sostener explotaciones familiares de carácter estable. Algunos autores opinan que el futuro del pastoreo trashumante parece asegurado, no sólo por la tradición cultural de esta práctica secular, sino porque las inversiones necesarias que entraña la intensificación ganadera obstaculizan el cambio desde el régimen extensivo al intensivo (Ruiz Pérez, 1988). En algunos casos, incluso, ciertas trashumancias son un fenómeno relativamente reciente debido a la imposibilidad de 
aprovechar los pastos de tierras comunales de muchos municipios españoles como consecuencia de las presiones repobladoras de los organismos forestales oficiales: según García Dory y Martínez Vicente (1988), las repoblaciones llevadas a cabo desde 1950 en nuestro país han alcanzado una superficie total próxima a los cuatro millones de hectáreas. Ello ha repercutido negativamente, como no podía ser de otra forma, en el desarrollo de la ganadería extensiva.

\section{Ganadería extensiva y ecosistemas naturales: dehesa y montaña}

La dehesa, que se extiende por el suroeste español, constituye una explotación compleja que logra mantener en activo extensos territorios de difícil puesta en cultivo. Fundamentalmente, la dehesa es un aprovechamiento ganadero surgida tras la degeneración del encinar y que coexiste también con los usos agrícolas y forestales. No es de extrañar, por tanto, que algunos autores consideren a la dehesa como el equilibrio casi perfecto del hombre con la naturaleza, una intervención humana sobre el bosque mediterráneo original consistente en aclarar la arboleda para dar como resultado un pastizal fácilmente aprovechable por el ganado con árboles aislados. Las importantes limitaciones que impone la dehesa a los cultivos, debido a que se localizan en terrenos pobres en materia orgánica, son superadas por la explotación ganadera extensiva, que contribuye al mantenimiento de la fertilidad del suelo y al desarrollo de los pastos mediante el estercolado.

La explotación es, de alguna manera, un aprovechamiento equilibrado de los recursos naturales. La evolución no se ha dirigido siempre hacia lo que se podría definir como estabilidad ecológica, ya que la disminución en algunos casos de la carga ganadera ha provocado la invasión de la dehesa por parte del matorral, y en otros lo que se ha producido es un excesivo laboreo en unos suelos que no admiten cultivos permanentes. El problema que subyace en el fondo de esta evolución ha sido la falta de rentabilidad de la ganadería extensiva tradicional. Algunas transformaciones, bien encauzadas, han superado ya esa situación en determinadas dehesas, como la repoblación de encinas y alcornoques, la mejora de los pastos naturales, el cerca-

$$
-133-
$$


miento de parcelas, la construcción de diferentes instalaciones para el ganado o la introducción del regadío en pequeñas zonas de la finca para producir verde y heno. El aumento de la carga en las explotaciones, por no citar las repercusiones que ello tendría sobre el empleo local y el medio natural, sería una de las consecuencias más importantes.

La modernización, por tanto, constituye un aspecto fundamental en una explotación como la dehesa que se caracteriza por la gran propiedad, lo que ha permitido no sólo la rotación de los cultivos y la consiguiente recuperación del suelo, sino también mantener una ganadería considerable pese a unas densidades ganaderas relativamente bajas.

La ganadería en zonas de montaña es otro punto importante en relación con el aprovechamiento de los ecosistemas naturales. La ganadería extensiva en estas áreas constituye un factor evidente de estabilización, sobre todo si se lleva a cabo un uso ordenado de los pastos, no hay un exceso de carga ganadera o la situación opuesta de falta de pastoreo suficiente. La mayoría de los autores consideran a los pastos de montaña como un resultado parcial de la acción reguladora del ganado sobre la vegetación, lo que hace posible su utilización en forma de recurso natural renovable.

Los problemas que rodean a esta ganadería son considerables en la actualidad. En primer lugar, no suele existir una base territorial asegurada, incidiendo negativamente la excesiva fragmentación de las explotaciones y la existencia de numerosas situaciones jurídicoadministrativas. Tampoco hay que olvidar un fenómeno típico de las montañas y sus repercusiones sobre las explotaciones ganaderas extensivas: la despoblación, ya que ha provocado la disminución de las cargas pastantes, el desequilibrio ecológico en numerosos sectores y la reducción de los ganados ovino, mular y caballar. La producción de pastizales presenta, además, fuertes oscilaciones a lo largo del año, con un mínimo invernal y un máximo estival. Esto condiciona el aprovechamiento de los pastos e impide completar el ciclo anual de la alimentación animal. Como solución se ha recurrido frecuentemente a las trasterminancias para aprovechar la variabilidad que ofrecen los límites altitudinales en relación con la vegetación natural. Las 
trashumancias, con recorridos de mayor distancia, también se han practicado desde las áreas de montaña a los pastos de invernada en tierras bajas.

Consecuencia de todos estos problemas es que se están dando una serie de tendencias que reorientan el aprovechamiento ganadero extensivo de montaña. En primer lugar destaca la disminución del ganado lanar, a veces hasta llegar casi a la desaparición (Pirineo Central). Esta circunstnacia provoca que no se utilicen los pastos de altura localizados por encima de los 1.600-1.800 metros, tendiendo hacia niveles progresivamente menos productivos. En oposición al descenso del ganado menor, se ha registrado un incremento considerable del ganado vacuno, pudiendo definir, incluso, a la explotación agraria de montaña como esencialmente ganadera de vacuno, tras una reorientación económica que ha supuesto el abandono del ganado extensivo y la tendencia generalizada hacia la producción láctea. Este aumento se ha dado sobre todo en las montañas más húmedas (Cordillera Cantábrica) y en las zonas de mayor descenso demográfico. En el primer caso porque se produce la renovación y el crecimiento rápidos del pasto, $\mathrm{y}$ en el segundo porque el vacuno presenta pocas dificultades en cuanto a la vigilancia del ganadero, lo que es muy importante en momentos de emigración.

Otra tendencia ha sido la marginación de las razas autóctonas de montaña como consecuencia de la intensificación de las explotaciones mediante razas importadas. Un dato significativo es el descenso experimentado por el ganado vacuno autóctono de montaña desde 1955 a 1991, pasando a 51.906 a 30.500 efectivos entre ambas fechas. La intensificación se ha llevado a cabo sin tener en cuenta los numerosos limitantes de que disponen las áreas de montaña, que son territorios de condiciones muy particulares de explotación. Las modificaciones sobre un sistema secularmente consolidado pueden alterar otros aspectos de la producción y, además, como pocas veces se han aplicado en su totalidad, han acabado por generar una acusada diversidad de sistemas y estrategias de producción, no muy definidos y de resultados mediocres.

En España, debido a la diversidad de los ecosistemas regionales, el uso de las razas autóctonas garantiza un correcto aprovechamiento

$$
-135-
$$


de los recursos naturales renovables. Íntimamente ligadas al territorio, su protección supone el fomento de la ganadería extensiva en su conjunto, evitándose así ciertos problemas ecológicos derivados de un escaso pastoreo y asegurando unos recursos genéticos que pueden ser muy necesarios en el futuro. Además, los altos niveles de producción ganadera ya alcanzados permiten la diversificación de la oferta existente en la actualidad con producciones de calidad.

\section{El ganado de lidia}

El estudio de las ganaderías de reses bravas queda aquí justificado por su explotación económica en régimen claramente extensivo. El análisis geográfico cobra, pues, pleno significado por las razones ya comentadas y por su relación con las políticas agrarias, la evolución de las estructuras rurales y las transformaciones y cambios productivos de numerosas zonas en las que tradicionalmente se han asentado estos ganados. Se relaciona también con la promoción de razas autóctonas, ya que el ganado de lidia constituye un patrimonio ganadero peculiar a través de un proceso centenario de cría selectiva y representa una forma muy característica de ganadería ligada a la tierra, al medio natural y a la estructura de la propiedad.

El significado económico de estas reses, aparte del puramente lúdico de la lidia en las plazas, es muy importante, ya que supone el aprovechamiento de recursos naturales en zonas marginales para el cultivo, de difícil mecanización, de fuerte emigración o alejadas de los núcleos de población. Estas explotaciones, por tanto, constituyen un instrumento eficaz de ocupación de espacios peculiares y un ejemplo de la más pura fórmula de ganadería extensiva. Su interés llevó al Ministerio de Agricultura a catalogar a la raza de lidia dentro del grupo de razas autóctonas de fomento del Catálogo Oficial de Razas de Ganado de España.

Antes de pasar a comentar detenidamente los valores estadísticos de la distribución de las reses bravas, cabe señalar que estas ganaderías presentan, desde el punto de vista del emplazamiento, un marcado carácter ribereño y serrano y una clara adscripción a las zonas de dehesa o monte adehesado (Sánchez Belda y otros, 1980). 
Cuadro IV

DISTRIBUCIÓN PORCENTUAL DE LAS PARIDERAS

EN GANADERÍAS BRAVAS

\begin{tabular}{|c|c|c|c|c|}
\hline Mes & Cádiz & Salamanca & Madrid & Sevilla \\
\hline 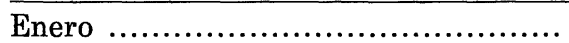 & 17,1 & 10,1 & 9,9 & 14,2 \\
\hline Febrero & 15,5 & 15,4 & 9,7 & 16,8 \\
\hline 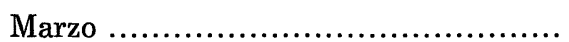 & 16,1 & 21,5 & 15,0 & 15,1 \\
\hline 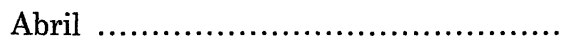 & 9,4 & 24,0 & 17,0 & 10,8 \\
\hline 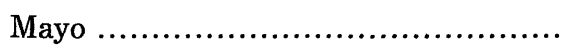 & 5,4 & 12,9 & 16,2 & 10,7 \\
\hline 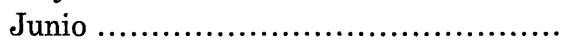 & 1,6 & 5,1 & 10,4 & 3,7 \\
\hline 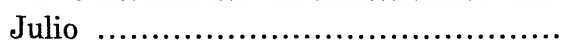 & - & - & 0,1 & 8,4 \\
\hline 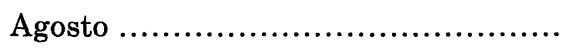 & - & - & 0,1 & 0,5 \\
\hline Septiembre & 2,8 & 0,2 & 1,5 & 1,4 \\
\hline 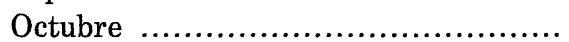 & 6,5 & 1,6 & 5,4 & 2,8 \\
\hline Noviembre $\ldots \ldots \ldots \ldots \ldots \ldots \ldots \ldots \ldots \ldots \ldots$ & 12,8 & 3,3 & 7,4 & 9,7 \\
\hline 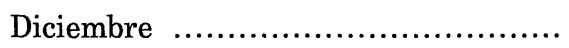 & 12,4 & 5,5 & 7,0 & 11,1 \\
\hline
\end{tabular}

Fuente: Sánchez Belda y otros, 1980.

En relación con el carácter ribereño, estas explotaciones se ubican tanto en los márgenes de los grandes ríos como en los regatos serranos y arroyos de tierras altas. Dentro de las cuencas fluviales, estos ganados ocupan áreas marginales, es decir, las cabeceras de los ríos o las tierras bajas de las desembocaduras. En Andalucía existe una persistente predisposición a localizarse en las proximidades de las marismas del Guadalquivir, en donde la lluvia suficiente y la temperatura adecuada permiten el desarrollo de pastos de buena calidad. Otro sector andaluz es Sierra Morena, zona alta opuesta a las explotaciones de las marismas y tradicionalmente dedicada a la cría de toro de lidia. Las explotaciones en zonas de campiña son escasas debido a la alta rentabilidad alcanzada con diversos cultivos agrícolas.

Muchas vegas han perdido su condición de criadoras de ganado bravo como consecuencia de traslados hacia cotas más elevadas y coincidiendo en la actualidad el asentamiento de estas ganaderías con los ríos de montaña. En efecto, muchos factores pueden actuar en el retroceso o desplazamiento de estas ganaderías, particularmente sensibles a las situaciones territoriales en las que se desenvuelven 
o motivado por el aprovechamiento de los pastos. En muchas zonas, la colonización agrícola, la urbanización o la puesta en marcha de áreas de regadíos han provocado un desplazamiento constante de los ganados de lidia hacia espacios menos congestionados y generalmente serranos, aunque sin afectar a nivel nacional al número total de efectivos.

Los traslados temporales, con la intención de aprovechar los pastos, han sido una constante de estas explotaciones extensivas. Menos frecuentes los desplazamientos de largo recorrido, los movimientos son generalmente de carácter trasterminante entre dehesas de municipios próximos de un mismo propietario. Los pastos mejores, más nutritivos y mejor aprovechados por el ganado bravo, son los de primavera, coincidiendo con la primera alimentación natural de los becerros nacidos en invierno. Los pastos naturales constituyen un factor determinante del sistema de explotación, ya que esa sumisión establece un calendario de partos y una gradación de disponibilidades para la lidia.

Estos datos ponen de manifiesto la dependencia y servidumbre del proceso reproductivo a las limitaciones del medio, nota característica de cualquier ganadería en régimen extensivo. En las zonas de clima suave se dan parideras tempranas, muy en relación con inviernos benignos y primaveras adelantadas. Por el contrario, las parideras tardías suponen una defensa contra las temperaturas del invierno y primaveras retrasadas. Esto sitúa a las ganaderías del sur peninsular en situación de relativa ventaja.

En relación con la distribución de los efectivos de ganado de lidia, se ha utilizado como fuente de información el Censo de la Ganadería Española del Ministerio de Agricultura, en donde aparece bien especificada la raza de lidia por provincias y Comunidades Autónomas. Manejando, además, las reseñas estadísticas provinciales se puede obtener información a nivel municipal para todas las provincias con efectivos. Los datos irán referidos a 1986, último año para el que se dispone de información estadística, ya que con posterioridad no se ha publicado ningún dato al respecto. Los Anuarios de Estadística Agraria, en la parte correspondiente al Censo Ganadero, ofrecen información sobre el número de terneros herrados de ganado de lidia 
y el número de ganaderías inscritas y su distribución asociativa a la Unión de Criadores de Toros de Lidia, a la Asociación Nacional de Ganaderos de Lidia, a los Ganaderos de Lidia Unidos y a la Asociación Española de Reses Bravas, pero sin un desglose pormenorizado de los efectivos totales.

El ganado bravo se distribuye por un total de 32 provincias españolas, lo que significa una dispersión aceptable de la raza, sobre todo si se compara con la distribución geográfica de otras razas bovinas autóctonas. El ganado de lidia se corresponde principalmente con las zonas del centro, oeste y sur peninsulares, siendo las otras localizaciones secundarias o marginales. Lo más llamativo es la ausencia absoluta de ganado bravo en la España verde, es decir, Galicia, Asturias, Cantabria y País Vasco. Otra nota característica es la ausencia en la España insular, ya que ni en Baleares ni en Canarias se encuentran efectivos de reses bravas. En toda la costa mediterránea, desde Cataluña a Murcia, el número de cabezas es bastante escaso, concentrándose prácticamente en su totalidad en las provincias de Castellón y Tarragona.

En 1986 había en España un total de 119.971 cabezas de ganado de lidia, cifra muy significativa en cuanto a razas bovinas autóctonas, pero que sólo representa el $2,4 \%$ de todo el ganado bovino en el país. Este porcentaje ha disminuido progresivamente desde 1950 debido al masivo incremento de las razas importadas, aumentando de forma espectacular el censo vacuno en su conjunto. En cualquier caso, como señalan García Dory y Martínez Vicente (1988), el ganado de lidia es de las pocas razas autóctonas que han incrementado sus efectivos.

Las variaciones del censo de toros bravos se deben a las condiciones climáticas y a las estrategias del propio ganadero, de manera que la reducción próxima a las 9.000 reses de 1982 a 1986 se explica por las consecuencias de la sequía que afectó al país desde 1980 , lo que motivó una merma retardada de los efectivos totales. Si en 1981 se herraron un total de 18.429 terneros, según el Anuario de Estadística Agraria, en 1982 lo hacen 14.701, no recuperándose la cifra inicial hasta 1988, en que se hierran un total de 18.525 terneros. Por otra parte, los descensos más significativos se producen en las regio- 
Cuadro V

EFECTIVOS DE RESES BRAVAS EN ESPAÑA

\begin{tabular}{|c|c|c|c|}
\hline CC.AA. & 1986 & $\%$ & Variación 1982-1986 \\
\hline Navarra & 2.365 & 2,0 & +1.548 \\
\hline La Rioja ................................. & 222 & 0,2 & +20 \\
\hline Aragón . ............................... & 3.710 & 3,1 & +1.846 \\
\hline Cataluña ................................ & 1.063 & 0,9 & +288 \\
\hline Castilla y León .... & 26.477 & 22,1 & -6.359 \\
\hline Madrid ................................. & 5.556 & 4,6 & -38 \\
\hline Castilla-La Mancha ................... & 10.490 & 8,7 & -3.690 \\
\hline Comunidad Valenciana ............... & 2.778 & 2,3 & +676 \\
\hline Extremadura ............................. & 10.735 & 8,9 & -1.990 \\
\hline Andalucía ............................ & 56.575 & 47,2 & -839 \\
\hline 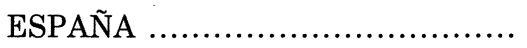 & 119.971 & 100 & -8.639 \\
\hline
\end{tabular}

FUENTE: MAPA, Censo de la Ganadería Española, 1982 y 1986, y elaboración propia.

nes de menores precipitaciones y donde la sequía tuvo efectos más negativos, como en Castilla y León, Castilla-La Mancha y Extremadura. Esto demuestra la gran sensibilidad de estas ganaderías a las condiciones naturales del territorio.

A nivel provincial destacan Salamanca, Sevilla y Cádiz, seguidas de Jaén y Toledo. En la figura 2 se representa la distribución del ganado de lidia en España, diferenciando tres áreas distintas: el área principal, que abarca el centro y oeste peninsulares, incluye las provinciais de Salamanca, Cáceres, Badajoz, Sevilla, Cádiz, Jaén, Madrid y Toledo; estas provincias son las que cuentan con mayores efectivos para la lidia a nivel nacional, con cifras muy superiores a las del resto de provincias. El área secundaria engloba aquellas provincias próximas a las primeras o las que constituyen de forma aislada puntos de relativa concentración: Zamora, Valladolid, Avila, Segovia, Huelva, Córdoba, Granada, Ciudad Real, Albacete, Valencia, Castellón, Tarragona, Teruel, Zaragoza, Logroño y Navarra. Por último, el área marginal agrupa a las provincias con mínimos efectivos y que no constituyen lugares de asiento tradicional de vacadas bravas; su distribución es muy dispersa por el territorio nacional: Palencia, Burgos, Málaga, Cuenca, Alicante, Guadalajara, Huesca y Barcelona. El cuadro 6 adjunto resume el censo de ganado de lidia en cada una de esas tres áreas establecidas. 


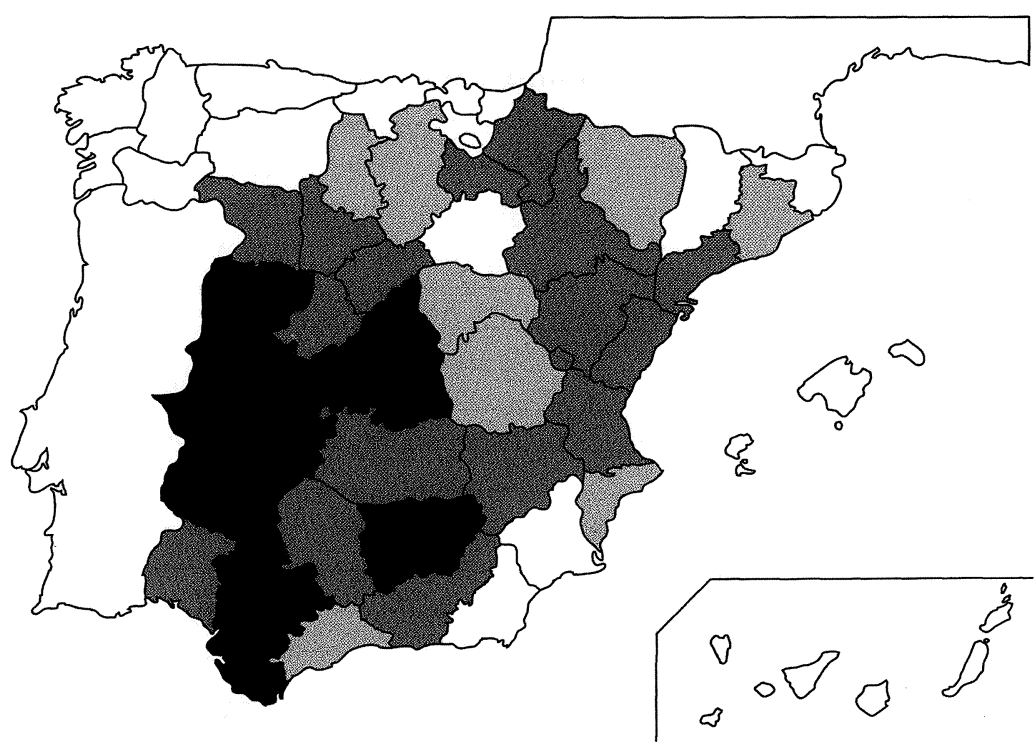

Figura 2.- Distribución del ganado de lidia en España. 1986. 1. área principal; 2. id. secundaria; 3. id. marginal.

\section{CuAdro VI}

ÁREAS DE DISTRIBUCIÓN DEL GANADO DE LIDIA

\begin{tabular}{lccc} 
Área & Número provincias & Total ganado lidia & \% \\
\hline Area principal $\ldots \ldots \ldots \ldots \ldots \ldots \ldots \ldots \ldots \ldots$ & 8 & 94.082 & 78,4 \\
Area secundaria $\ldots \ldots \ldots \ldots \ldots \ldots \ldots \ldots \ldots \ldots$ & 16 & 25.525 & 21,3 \\
Area marginal $\ldots \ldots \ldots \ldots \ldots \ldots \ldots \ldots \ldots \ldots$ & 8 & 364 & 0,3 \\
Total $\ldots \ldots \ldots \ldots \ldots \ldots \ldots \ldots \ldots \ldots \ldots \ldots \ldots \ldots$ & 32 & 119.971 & 100
\end{tabular}

Fuente: MAPA, Censo de la Ganadería Española 1986, y elaboración propia.

\section{Crisis y transformación: tendencia hacia la explotación intensiva}

La ganadería extensiva no sólo es sensible a las condiciones del territorio en que se desenvuelve, sino también a las circunstancias socioeconómicas generales. El desarrollo económico ha traído consigo importantes cambios en zonas tradicionalmente dedicadas a la producción extensiva de animales. Las nuevas demandas alimentarias, unidas a un crecimiento que parece que ha consagrado definiti- 
vamente la producción capitalista en las explotaciones agrarias, han incentivado la intensificación de la ganadería mediante la introducción de razas selectas, el desplazamiento de la mano de obra hacia la industria y el sector servicios, el abandono de amplias áreas rurales y de los sistemas tradicionales de explotación, etc. En definitiva, se ha operado un proceso de cambio a favor de la ganaderia industrial e intensiva, es decir, de la ganadería no ligada al suelo.

La intensificación supone la modificación de los factores de producción. Aunque de forma general se habla de explotación intensiva, es más correcto referirse a distintos grados de intensificación de las explotaciones ganaderas, ya que la producción intensiva es un eslabón extremo que obvia los tramos intermedios en el proceso de mejora de los distintos índices productivos. Esta intensificación ha generado lo que algunos autores han definido como «crisis de la ganadería tradicional» (Rodríguez, Ruiz y Soria, 1980), así como un descenso espectacular de la ganadería en numerosas zonas del país, caracterizadas frecuentemente por una trabazón secular entre producción animal, tierra y recursos naturales.

La intensificación ganadera es resultado del incremento en los niveles de industrialización. El crecimiento de la renta eleva la demanda de productos, demanda que, por otra parte, requiere un abastecimiento regular y alejado de las fluctuaciones propias de todo sistema extensivo, que no se vincula con circuitos comerciales estables y de suficiente amplitud y alcance. La sustitución de las explotaciones tradicionales por otras sin base territorial, a las que se podría calificar de industrializadas, está servida.

Este proceso ha caracterizado el desarrollo ganadero en España aproximadamente desde 1960. El fin del período de autarquía impulsó la puesta en práctica de una serie de recomendaciones elaboradas por una misión del Banco Mundial y de la FAO, que llega a nuestro país en 1965. La orientación principal, según se desprende del «Informe del Banco Mundial y de la FAO sobre el desarrollo de la agricultura en España», publicado en 1967 por el Ministerio de Hacienda, era la de aumentar la producción ganadera, suponiendo la penalización inevitable de la ganadería extensiva y la dirección unilateral hacia la intensificación de la producción. 
CuAdro VII

EVOLUCIÓN DE LOS EFECTIVOS DE VACUNO EN ESPAÑA

\begin{tabular}{lcc} 
Año & \multicolumn{2}{c}{ Razas } \\
\cline { 2 - 3 } & Extranjeras & Autóctonas \\
\hline 1955 & 442.054 & 1.260 .988 \\
1970 & 1.095 .283 & 1.214 .484 \\
1978 & 1.582 .420 & 929.146 \\
1986 & 1.914 .169 & 819.628
\end{tabular}

FuENTE: MAPA, Anuario de Estadistica Agraria, 1988.

La evolución de los efectivos problacionales de las distintas especies es muy significativa de la situación, siendo las razas autóctonas las que han sufrido en mayor medida las consecuencias de la crisis del sistema extensivo. La especie bovina disponía en 1955 de un total de 442.054 cabezas pertenecientes a razas extranjeras. Como la intensificación se ha basado en razas importadas, en 1986 el número de cabezas de vacuno de razas extranjeras se eleva a casi dos millones. Es decir, el aumento ha sido espectacular y, con ello, el de los sistemas intensivos de producción animal. Las razas autóctonas, por el contrario, han mantenido la tendencia inversa, con una reducción para el mismo período de casi medio millón de cabezas, pasando de 1.260 .988 en 1955 a 819.628 en 1986. Las razas cantábricas, sobre todo las que se localizan en áreas montañosas, como la Asturiana de Montaña y la Tudanca, han sido las más afectadas. En otros casos, se ha resistido mejor la tendencia hacia la intensificación de las explotaciones, de forma que las razas Retinta y Lidia han aumentado considerablemente. Se puede afirmar, por tanto, que las razas propias de las zonas adehesadas del sur y oeste peninsulares han resistido mejor la extendida producción intensiva.

La intensificación llevada a cabo en el ganado bovino y porcino ha motivado un gran incremento de la producción de carne en nuestro país. El principal protagonista del despegue productivo de la ganadería española en las últimas décadas ha sido el ganado porcino, que ha pasado de producir 179.589 toneladas de carne en 1949 a 1.788 .848 en 1990 , lo que casi significa multiplicar por diez las cifras iniciales. Los cambios no sólo son cuantitativos, sino también cualita- 
CuAdro VIII

PRODUCCIÓN DE CARNE EN ESPAÑA (TM)

\begin{tabular}{lrrrr} 
Año & Bovino & Ovino & Caprino & Porcino \\
\hline 1940 & 83.416 & 53.686 & 12.822 & 179.589 \\
1950 & 91.181 & 66.024 & 13.697 & 140.962 \\
1960 & 159.579 & 109.941 & 11.744 & 257.893 \\
1970 & 308.160 & 127.093 & 12.689 & 491.749 \\
1980 & 421.549 & 168.389 & 13.682 & 1.182 .308 \\
1990 & 513.898 & 217.396 & 16.417 & 1.788 .848
\end{tabular}

FuENTE: MAPA, Anuario de Estadistica Agraria, varios años.

tivos. Para el aumento registrado ha habido que importar casi por completo la cabaña porcina y aclimatarla en nuestro país en granjas de alta tecnología. Ello ha provovocado un desplazamiento de la cabaña desde el oeste hacia el Levante, sobre todo Cataluña, como principal foco productor. La evolución en el bovino también ha sido considerable.

Pese al crecimiento de la producción de carne, el consumo ha acelerado un comercio exterior deficitario que se sitúa en 153.645 toneladas en 1990 (239.111 Tm. importadas y 85.466 Tm. exportadas). Es a partir de 1980 cuando aumentan bastante las importaciones,

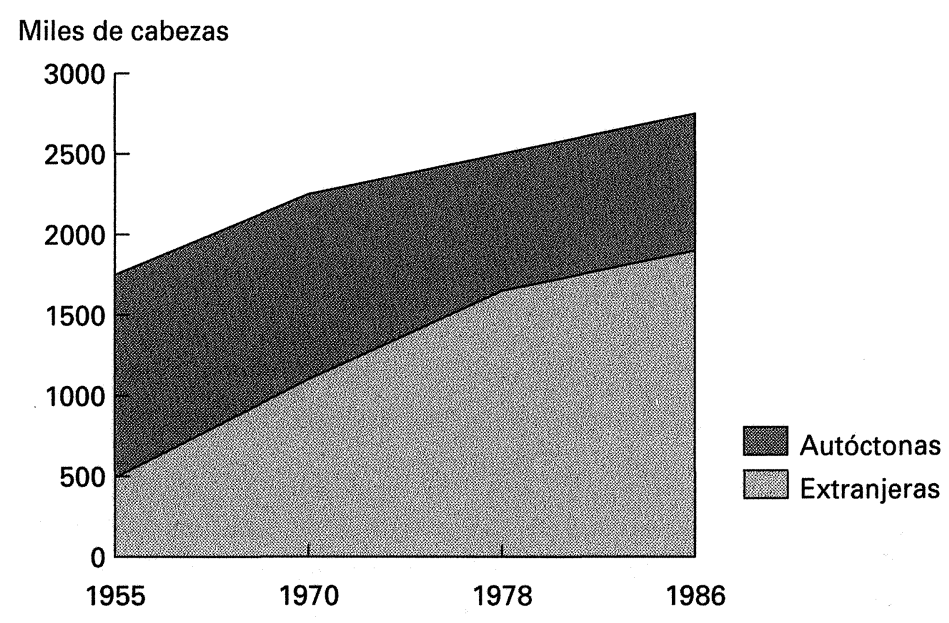

FigURA 3.-Razas vacunas en España autóctonas e importadas 
tras una reducción constante desde 1970, lo que ha podido incentivar aún más la intensificación ganadera para cubrir las necesidades nacionales y agravar la ya profunda crisis por la que atraviesa la ganadería extensiva.

Aunque el cambio hacia una ganadería intensiva y estante comienza a finales del siglo xIx, sólo se acelera a partir de la década de 1960 , como ya se ha comentado, en que se incorporan nuevas técnicas, se abre el mercado español a las materias primas importadas con destino a la ganadería, aumenta el nivel de vida, etc. En primer lugar, se generalizan los sistemas ganaderos industriales en la producción de carne de cerdo. En el bovino y ovino, la transformación hacia un mayor grado de intensificación se realiza sobre todo en la década de 1970 (Sobrino Igualador y otros, 1981). Sólo recientemente se ha percibido la importancia que suponen los sistemas de tipo extensivo y se ha procurado su potenciación, no sólo para corregir la tendencia regresiva de la ganadería en numerosas zonas del país, como las áreas de montaña, ahora infrautilizadas por el descenso ganadero, sino también para reducir el fuerte déficit de las últimas décadas en la Balanza de Pagos Agrícola por las importaciones masivas de piensos compuestos. La base alimenticia de la ganadería intensiva, los piensos compuestos, crea una fuerte dependencia del exterior, muy elevada en España en comparación con otros países comunitarios y en relación también con los recursos naturales disponibles.

El sometimiento del campo a la industria tras la generalización del desarrollo económico está en la misma base de la crisis de la ganadería extensiva, ya que con la industrialización ha venido la despoblación de muchas áreas rurales, el encarecimiento de la mano de obra, la extensión de la mecanización, la intensificación de los sistemas productivos para atender una demanda en constante crecimiento, etc. Las repercusiones han sido importantes en la ganadería extensiva como consecuencia de una serie de características propias de la producción ganadera ligada a la tierra, como la tradicional disponibilidad de un mano de obra abundante y de difícil sustitución, la poca capitalización de las explotaciones y una producción basada en una ganadería poco mejorada y de mediocre rentabilidad. No hay que olvidar tampoco como causa importante de la crisis las adversidades climatológicas y la escasez de pastos en determinados períodos del año.

$$
-145-
$$


La intensificación ha sido la alternativa mayoritaria a la crisis, pero en aquellas zonas de menor desarrollo económico no se ha manifestado aún con la suficiente claridad, por lo que se podrían ver beneficiadas por algunas medidas reorientadoras que han pretendido potenciar los sistemas de tipo extensivo y las razas autóctonas. Hay que citar, en este sentido, el Real Decreto 464/1979, de 2 de febrero, Orden de 31 de enero de 1979 y, más recientemente, la nueva política agraria comunitaria, en la que se fijan altas primas para apoyar a las ganaderías extensivas o semiextensivas.

A partir del citado Real Decreto, sobre fomento en España de la ganadería extensiva y en zonas de montaña, la política ganadera persigue un mejor aprovechamiento de los recursos naturales como forma de reducir las importaciones de productos agrícolas destinados a la alimentación animal. Las acciones encaminadas a este impulso se refieren, en primer lugar, a la necesaria consolidación de explotaciones potencialmente viables con mejora de su productividad y racionalización de su proceso productivo. Y, en segundo lugar, se considera imprescindible la movilización de recursos en zonas subutilizadas y deprimidas y de tradicional producción animal.

La Orden de 31 de enero de 1979, sobre fomento de las razas ganaderas autóctonas promocionables, contenía un programa de acción limitado a aquellas comarcas caracterizadas básicamente por la producción de ganado en régimen de pastoreo, siempre y cuando de esa forma se pudieran satisfacer al menos el $70 \%$ de las necesidades alimenticias del rebaño reproductor. Las explotaciones ganaderas acogidas a este programa recibirían en régimen de depósito un ejemplar cedido de raza catalogada por cada tres que adquirieran los titulares de las mismas.

En la reforma de la Política Agraria Comunitaria, el sector de vacuno es el que ha experimentado las mayores modificaciones, algunas de ellas encaminadas a potenciar la ganadería extensiva o semiextensiva y congelar la oferta. Esta reforma supone un fuerte revés para la ganadería intensiva en España, que aporta en torno al $80 \%$ de la carne de vacuno, ya que los cebaderos quedan excluidos de la práctica totalidad de las primas que se fijan para la ganadería ligada a la tierra. Para acceder a las primas (180 ECUs para bovinos ma-

$$
-146-
$$


chos pagaderos a los diez y veintidós meses de vida), la explotación debe tener una carga ganadera de $2 \mathrm{UGM} /$ hectárea, algo que no cumple la mayor parte de las explotaciones intensivas españolas. A esta prima se suma otra por extensificación de 30 ECUs cuando la carga ganadera sea de 1,4 unidades por hectáreas.

Estas medidas pueden potenciar en nuestro país una ganadería autóctona hasta ahora regresiva en muchas zonas pero que tradicionalmente han convertido en productivos unos espacios difícilmente aprovechables por otros usos, como las zonas de montaña, y ello mediante la utilización adecuada del medio natural. La promoción de esta ganadería no sólo diversifica la producción sino que se plantea como una respuesta válida a la excesiva dependencia que representan los sistemas ganaderos intensivos.

El ahorro de recursos destinados a la compra de piensos compuestos puede conllevar, por ejemplo, beneficios económicos estables para los ganaderos, independizándose de estrategias económicas diversas al aprovechar, a base de razas autóctonas, unos recursos renovables nada despreciables. En cualquier caso, la superación del desorden actual por el que atraviesa la ganadería extensiva obliga a una nueva concepción de la actividad que la haga más competitiva y que suponga la aplicación de técnicas variadas, la mejora de los pastizales, la modernización de las instalaciones, el cruce genético para incrementar la producción y la incorporación de ciertas medidas sanitarias. Sólo de esta forma, el futuro de la ganadería extensiva podría quedar garantizado.

\section{BIBLIOGRAFÍA}

AA.VV. (s.f.): La dehesa: un patrimonio que se pierde, Centro Castellano de Estudios Ambientales, Cuaderno núm. 1.

Abella, M. A., y otros (1988): «Sistemas ganaderos de montaña», Agricultura y Sociedad, 46, pp. 119-190.

Abellán, A., y Olivera, A. (1979): «La trashumancia por ferrocarril en España», Estudios Geográficos, 156-157, pp. 385-414.

Abreu i Pidal, J. M. (1981): «Variabilidad en las áreas de montaña», Revista de Estudios Agro-Sociales, 116, pp. 259-263.

Blas, C. de (1983): Producción extensiva de vacuno, Madrid, Ediciones Mundi-Prensa.

$$
-147-
$$


Fillat, F. (1980): «La explotación ganadera como estrategia para frenar la pérdida de fertilidad en montaña», Cuadernos de Investigación Geográfica, VI, 1-2, pp. 37-49.

- (1984): «Una forma de explotación tradicional de la montaña: la ganadería extensiva», III Curso de Ordenación del Territorio, Lérida, ETSIA.

GARCIA DE OTEYZA, L. (1981): «La ganadería española en la década de los 80», Revista de Estudios Agro-Sociales, 116, pp. 9-15.

García Dory, M. A. (1980): «La utilización de las razas autóctonas en los ecosistemas regionales, como factor de ahorro energético en la ganadería española», Agricultura y Sociedad, 15, pp. 115-162.

- y Martíz Vicente, S. (1988): La ganadería en España, Madrid, Alianza Editorial.

García Martín, P., y Sanchez Benito, J. M. (Comp.) (1986): Contribución a la historia de la trashumancia en España, Madrid, Ministerio de Agricultura, Pesca y Alimentación.

García Martín, P. (1990): El patrimonio cultural de las Cañadas Reales, Valladolid, Consejería de Cultura y Bienestar Social.

García Ruiz, J. M., y BaLcells, E. (1978): «Tendencias actuales de la ganadería en el Alto Aragón», Estudos Geográficos, 153, pp. 519-538.

IzquiERDo, J. A. (1977): «La trashumancia, de las dehesas extremeñas a los puertos de León», Agricultura, 537, pp. 101-104.

MarTín Bellido, M. (1989): «Producción animal en el suroeste español», en Pastos, forrajes y producción animal en condiciones extensivas, Badajoz-Elvas, Sociedad Española para el Estudio de los Pastos-Caja de Badajoz, pp. 309-333.

MARTín Galindo, J. L. (1966): «La dehesa extremeña como tipo de explotación agraria», Estudios Geográficos, 103, pp. 157-226.

Montoya Oliver, J. M. (1983): Pastoralismo mediterráneo, Madrid, ICONA.

Muslera Pardo, E., y Ratera García, C. (1978): «La mejora de pastos y el desarrollo de la ganadería extensiva enel suroeste español», Badajoz, $1 .{ }^{a}$ Reunión de la Red de Cooperación Europea para Pastos y Forrajes, 20 pp.

Muslera, E., y Montes, T. (1978): «Dehesas extremeñas. Pastos y cercas: sistemas diferidos de pastoreo», Agricultura, 552, pp. 289-292.

Olea Marquez de Prado, L.; Paredes Galan, J., y Verdasco Giralt, P. (1988): Mejora de pastos de la dehesa del suroeste de la Peninsula Ibérica, Madrid, Hojas Divulgadoras, 17, Ministerio de Agricultura, Pesca y Alimentación.

Ortega ValCÁRCEL, J. (1989): «La economía de montaña: una economía de equilibrio», Ería, pp. 115-128.

PARDo ABAD, C. J. (1987): «Las explotaciones ganaderas de reses bravas en las dehesas de El Escorial», Estudios Geográficos, 187, pp. 294-303.

PARDo ABAD, C. J. (1988): «El ganado bravo en Castilla-La Mancha: análisis de una actividad ganadera extensiva», en El espacio rural de Castilla-La Mancha, tomo I, Ciudad Real, Biblioteca de Autores y Temas Manchegos, pp. 227-237.

PARDo ABAD, C. J. (1994): «Ganadería extensiva y aprovechamiento de los ecosistemas naturales de montaña: evolución, crisis y transformación», Córdoba, Actas del VII Coloquio de Geografía Rural, pp. 254-260.

Puig Sales, R. (1981): «Ganadería y áreas de montaña», Revista de Estudios Agrosociales, 116, pp. 91-128.

Revilla Delgado, R., y Manrique Persiva, E. (1980): «La explotación de los puertos en el Valle de Gistain (Pirineo Oscense)», Trabajos del Instituto de Economia y Producciones Ganaderas del Ebro, 48, pp. 1-16. 
Rodriguez, M.; RuIz, J., y SorIA, R. (1980): «El desarrollo ganadero español: un modelo dependiente y desequilibrado», Agricultura y Sociedad, 14, pp. 165-193.

RuIz PÉREz, M. (1988): «Análisis económico de explotaciones ganaderas trashumantes», Revista de Estudios Agro-Sociales, 146, pp. 209-229.

SÁnchez Belda, A. (1977): «Ovejas, explotación extensiva», Agricultura, 537, pp. 23-25.

SÁnchez y SÁnchez-VALdePeñas, V. (1978): «Ganadería extensiva y recursos naturales», Agricultura, 552, pp. 274-278.

Sobrino IguAlador, F., y otros (1981): «Evolución de los sistemas ganaderos en España», Revista de Estudios Agro-Sociales, 116, pp. 17-90.

Vera Vega, A. (1986): Alimentación y pastoreo del ganado ovino, Córdoba, Universidad de Córdoba.

RESUMEN: La ganadería extensiva constituye un aprovechamiento ganadero claramente diferenciado de aquel otro basado en la intensificación de las producciones y desvinculado de bases territoriales concretas. Tradicional actividad económica en numerosas zonas españolas, en la actualidad padece una profunda crisis como consecuencia de la competencia planteada por la ganadería intensiva y la falta de capacidad de respuesta por parte de unos sistemas poco evolucionados y escasamente relacionados con circuitos comerciales de suficiente amplitud. Las salidas han sido diversas y los resultados no siempre positivos. Es necesaria la potenciación de la ganaderia extensiva con medidas diversas desde la Administración, posible en un momento en que se han alcanzado óptimos niveles productivos y podía ser interesante la oferta de producciones de elevada calidad procedentes de la ganadería extensiva, ligada directamente a territorios concretos y a la utilización de unos ecosistemas de considerables potencialidades.

Palabras Claves: Ganadería extensiva. Superficies pastables. Crisis. 